\title{
A SEARCH FOR RADIO EMISSION FROM TYPE Ia SUPERNOVAE
}

\author{
Nino Panagia, ${ }^{1,2}$ Schuyler D. Van Dyk, ${ }^{3}$ Kurt W. Weiler, ${ }^{4}$ Richard A. Sramek, ${ }^{5}$ \\ Christopher J. Stockdale, ${ }^{6}$ and Kimberly P. Murata ${ }^{7}$ \\ Received 2006 February 21; accepted 2006 March 29
}

\begin{abstract}
We present and discuss the radio observations of 27 Type Ia supernovae (SNe Ia), observed over two decades with the Very Large Array. No SN Ia has been detected so far in the radio, implying a very low density for any possible circumstellar material established by the progenitor, or progenitor system, before explosion. We derive $2 \sigma$ upper limits to a steady mass-loss rate for individual SN systems as low as $\sim 3 \times 10^{-8} M_{\odot} \mathrm{yr}^{-1}$, which argues strongly against white dwarf accretion via a stellar wind from a massive binary companion in the symbiotic star, an example of the "single-degenerate" scenario. However, a white dwarf accreting from a relatively low mass companion via a sufficiently high efficiency $(>60 \%-80 \%)$ Roche lobe overflow is still consistent with our limits. The "doubledegenerate" merger scenario also cannot be excluded.
\end{abstract}

Subject heading: binaries: close — binaries: symbiotic — radio continuum: stars — stars: mass loss — supernovae: general — white dwarfs

\section{INTRODUCTION}

Supernovae ( $\mathrm{SNe}$ ) are among the most energetic events in the universe. Determining the properties of the progenitor stars or stellar systems remains an important unsolved problem in astrophysics. Few SNe have had their progenitor stars directly identified, but the postexplosion radio emission from $\mathrm{SNe}$ provides insight into the nature of the progenitor stars and their last stages of evolution. The phenomenon of radio $\mathrm{SNe}(\mathrm{RSNe})$ has been best modeled by synchrotron emission resulting from the interaction of the SN shock with circumstellar material (CSM) established by pre-SN mass loss from the progenitor system before the explosion; likely from the progenitor itself, or possibly from the progenitor's companion in the case of a binary system (Chevalier 1982a, 1982b; Sramek et al. 1984; Weiler et al. 1986).

Although it has been generally accepted that Type Ia $\mathrm{SNe}$ (SNe Ia) result from the thermonuclear explosion of a white dwarf (WD) star (e.g., Nomoto et al. 1984; Branch et al. 1985), two fundamental questions remain: (1) Is the exploding WD of Chandrasekhar or sub-Chandrasekhar mass? (2) If the former, how does the exploding WD (typically $\sim 0.6 M_{\odot}$; e.g., Ritter \& Burkert 1986) accumulate enough mass to approach the Chandrasekhar limit of $\sim 1.4 M_{\odot}$ prior to explosion? In the so-called singledegenerate scenario (Nomoto et al. 1984), the source of the accreted material is provided by interaction with a nondegenerate companion, such as a low-mass main-sequence star, a subgiant, or a giant star (see the reviews by Branch et al. 1995 and Livio 2001). Under the "double-degenerate" scenario (Iben \& Tutukov

\footnotetext{
1 Space Telescope Science Institute, 3700 San Martin Drive, Baltimore, MD 21218; panagia@stsci.edu.

2 Istituto Nazionale di Astrofisica (INAF), Rome, Italy; and Supernova Ltd., Virgin Gorda, British Virgin Islands.

3 Spitzer Science Center, California Institute of Technology, Pasadena, CA 91125; vandyk@ipac.caltech.edu.

4 Naval Research Laboratory, Code 7213, Washington, DC 20375-5320; kurt.weiler@nrl.navy.mil.

${ }^{5}$ National Radio Astronomy Observatory, P.O. Box O, Socorro, NM 87801; dsramek@nrao.edu.

${ }^{6}$ Department of Physics, Marquette University, P.O. Box 1881, Milwaukee, WI 53201-1881; christopher.stockdale@marquette.edu.

79031 Ashmeade Drive, Fairfax, VA 22032; kpm1@cornell.edu.
}

1984; Webbink 1984) the explosion is triggered by the merger of two degenerate stars, such as WDs or neutron stars.

The nature of SN Ia progenitors has become even more important in the last decade because of their fundamental importance for cosmology (e.g., Riess et al. 1998; Perlmutter et al. 1999; Perlmutter \& Schmidt 2003). Unfortunately, we are in the embarrassing situation that, even as confidence in the astounding new cosmological results increases, we still have little knowledge of the physical origins of the luminous SN Ia explosions on which these results are at least partly based.

Observations of SNe Ia in the radio can provide a powerful constraint on the Chandrasekhar mass explosion mechanism in that the mass exchange required for the single-degenerate scenario should establish an enhanced CSM density, however tenuous, near the SN progenitor, while the double-degenerate scenario should not, unless the two coalescing WDs are in a common envelope (see Livio 2001). Thus, radio detection of the blast wave interaction with the CSM would not only support the singledegenerate scenario, but also provide information as to its extent, density, and structure.

To test the single-degenerate case for a symbiotic star progenitor system (in which a red giant donates mass to the WD via a wind), Boffi \& Branch (1995) modeled putative fast-evolving radio light curves for the SN Ia 1986G through analogy with the radio light curves for the SN Ib 1983N (Sramek et al. 1984; Weiler et al. 1986). From radio observations of SN $1986 \mathrm{G}$ conducted 1 week before optical maximum (i.e., early enough to adequately test the Boffi \& Branch prediction), Eck et al. (1995) concluded, based on a lack of detected radio emission, that this SN probably did not arise from a symbiotic star system. Eck et al. stressed that further searches for prompt radio emission from other SNe Ia were clearly necessary to test this and other models.

Numerous radio observations have been obtained for $27 \mathrm{SNe}$ Ia using the Very Large Array (VLA) ${ }^{8}$ as part of a SN monitoring program we have been conducting for more than two decades. In our program we have considered only extragalactic $\mathrm{SNe} \mathrm{Ia}$, and

\footnotetext{
${ }^{8}$ The VLA telescope of the National Radio Astronomy Observatory is operated by Associated Universities, Inc. under a cooperative agreement with the National Science Foundation.
} 
TABLE 1

Observed SNe

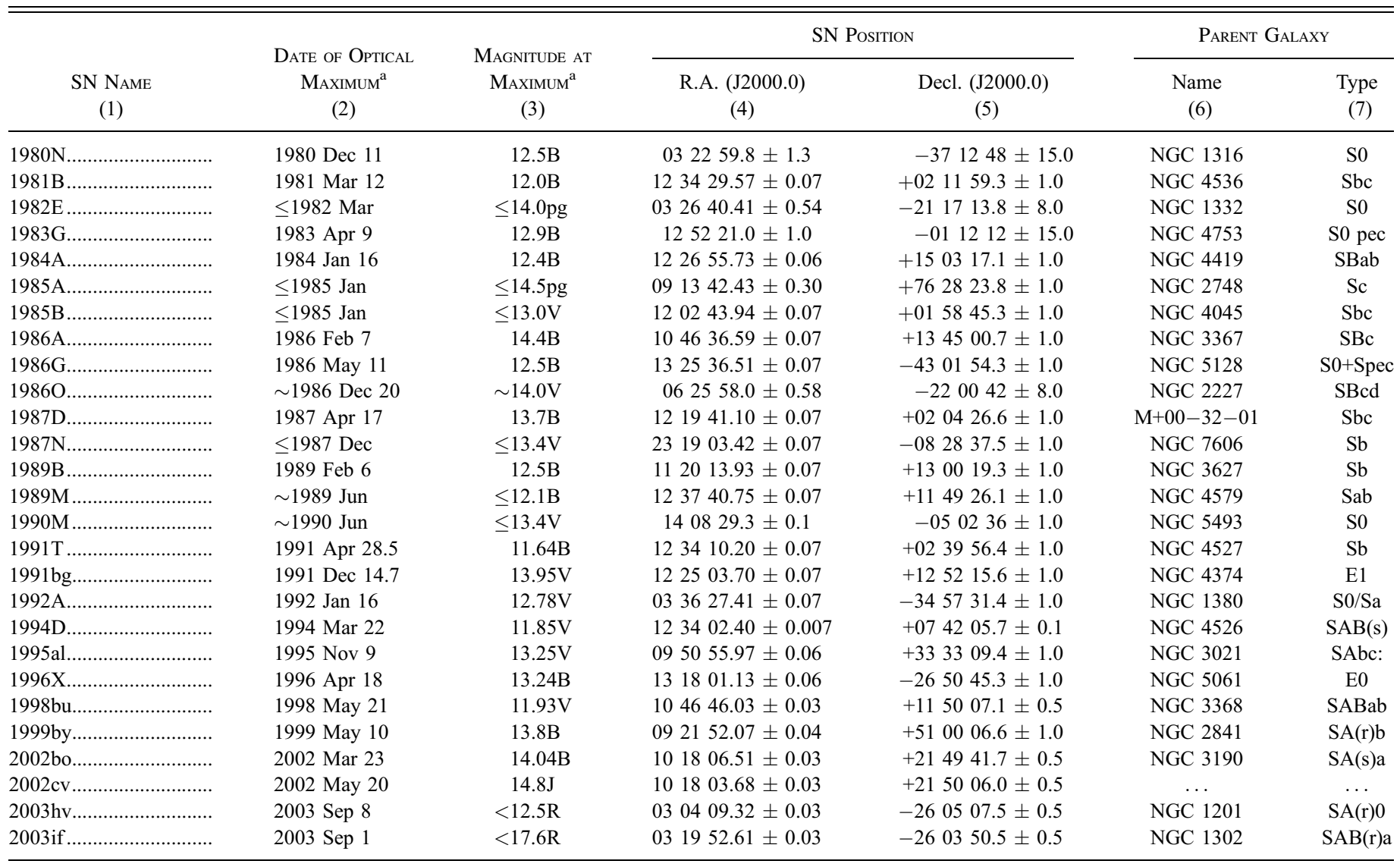

Nоте.- Units of right ascension are hours, minutes, and seconds, and units of declination are degrees, arcminutes, and arcseconds.

${ }^{a}$ Date and magnitude at discovery if the information is not available for the SN maximum.

we have not included any of the historical SNe Ia that have occurred in the Milky Way. Earlier results of SN Ia observations were presented by Weiler et al. (1986, 1989) and Weiler \& Sramek (1988). Unlike Type II and Type Ib/c SNe, no SN Ia has yet been detected as a radio emitter, even when observed quite promptly after explosion (e.g., SNe 1981B and 1980N; Weiler et al. 1986, 1989) or quite nearby (e.g., SN 1972E; Cowan \& Branch 1982, 1985; Weiler et al. 1989). Here, we present our new results with improved sensitivity and time and frequency sampling, and discuss the possible implications for the nature of the SN Ia progenitors.

\section{RADIO OBSERVATIONS}

The sample of observed SNe Ia consists of those generally with $m \leq 14$ mag occurring between 1982 and 2002. The sample includes the "Branch normal" SNe Ia 1992A (e.g., Kirshner et al. 1993) and 1994D (e.g., Richmond et al. 1995), as well as the overly luminous SN 1991T (e.g., Filippenko et al. 1992a; Schmidt et al. 1994), the subluminous SN 1991 bg (e.g., Filippenko et al. 1992b; Leibundgut et al. 1993), and the peculiar SN 1986G (e.g., Phillips et al. 1987). Two SNe, 2002 bo (Szabo et al. 2003) and $2002 \mathrm{cv}$ (Di Paola et al. 2002), occurred within a few months in the same host galaxy, NGC 3190.

The SNe were observed with the VLA in a number of array configurations at primarily $6 \mathrm{~cm}$ wavelength $(4.8 \mathrm{GHz})$, although observations for several objects were also made at $20 \mathrm{~cm}(1.4 \mathrm{GHz})$, $3.6 \mathrm{~cm}(8.4 \mathrm{GHz}), 2 \mathrm{~cm}(15 \mathrm{GHz}), 1.3 \mathrm{~cm}(22 \mathrm{GHz})$, and $0.7 \mathrm{~cm}$ $(43 \mathrm{GHz})$. The techniques of observation, editing, calibration, and error estimation are described in previous publications on the radio emission from $\mathrm{SNe}$ (e.g., Weiler et al. 1986, 1990, 1991). For the SN 1986G in NGC 5128 observations, the presence of the very radio bright galaxy nucleus Centaurus A required the additional analysis steps of first self-calibrating on and then removing from the $u-v$ data the components of the bright radio galactic nucleus, before producing the final maps of the SN field.

The SNe Ia that were observed are listed in Table 1. These include observations already described in Weiler et al. (1986, 1989). Column (1) lists the SN name, columns (2) and (3) list the date and magnitude at optical maximum (if available; if not, the date and magnitude at discovery are listed), and columns (4) and (5) give the right ascension (R.A.) and declination (decl.) in the epoch $\mathrm{J} 2000.0$ coordinates that were used for the radio observations. Columns (6) and (7) list the parent galaxy name and Hubble type. The radio results are listed in Table 2. Column (1) of that table lists the SN name, and column (2) the date of observation. Column (3) lists the estimated age of the SN in days after explosion, and column (4) lists the VLA configuration of the observation. Columns (5)-(10) list the measured rms $(1 \sigma)$ error, in mJy, in the resulting maps at $20,6,3.6,2,1.3$, and $0.7 \mathrm{~cm}$ wavelengths, respectively.

In Figure 1, we present the $2 \sigma$ spectral luminosity upper limits for SNe Ia at the wavelengths of 20,6,3.6, and $2 \mathrm{~cm}$. The data were rather sparse at $1.3 \mathrm{~cm}$ (1 point) and $0.7 \mathrm{~cm}$ (1 point), so those two frequencies are not shown. In Figure 2 we show the limits for two of the SNe Ia (SN 1989B and SN 1998bu) that were particularly well observed. None of the SNe Ia were detected as a 
TABLE 2

Observations

\begin{tabular}{|c|c|c|c|c|c|c|c|c|c|}
\hline \multirow[b]{2}{*}{$\begin{array}{c}\text { SN } \\
\text { NAME } \\
(1)\end{array}$} & \multirow[b]{2}{*}{$\begin{array}{l}\text { Observation } \\
\text { Date } \\
(2)\end{array}$} & \multirow[b]{2}{*}{$\begin{array}{c}\mathrm{AGE}^{\mathrm{a}} \\
\text { (days) } \\
(3)\end{array}$} & \multirow[b]{2}{*}{$\begin{array}{l}\text { VLA } \\
\text { ConfIG. } \\
\quad(4)\end{array}$} & \multicolumn{6}{|c|}{ MAP rms $(1 \sigma)$} \\
\hline & & & & $\begin{array}{c}\sigma_{20} \\
(\mathrm{mJy}) \\
(5)\end{array}$ & $\begin{array}{c}\sigma_{6} \\
(\mathrm{mJy}) \\
(6)\end{array}$ & $\begin{array}{c}\sigma_{3.6} \\
(\mathrm{mJy}) \\
(7)\end{array}$ & $\begin{array}{c}\sigma_{2} \\
(\mathrm{mJy}) \\
(8)\end{array}$ & $\begin{array}{c}\sigma_{1.3} \\
(\mathrm{mJy}) \\
(9)\end{array}$ & $\begin{array}{c}\sigma_{0.7} \\
(\mathrm{mJy}) \\
(10)\end{array}$ \\
\hline 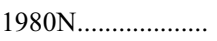 & 1981 Feb 3 & 72 & A & $\ldots$ & 0.20 & $\ldots$ & $\ldots$ & $\ldots$ & $\ldots$ \\
\hline \multirow[t]{10}{*}{ 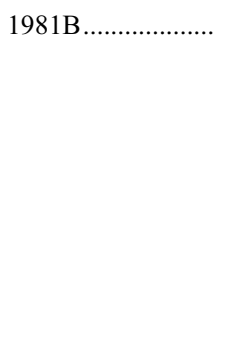 } & 1981 Mar 11 & 18 & A & $\ldots$ & 0.10 & $\ldots$ & $\ldots$ & $\ldots$ & $\ldots$ \\
\hline & 1981 Apr 9 & 46 & A & $\ldots$ & 0.13 & $\ldots$ & $\ldots$ & $\ldots$ & $\ldots$ \\
\hline & 1981 May 14 & 82 & $\mathrm{~B}$ & $\ldots$ & 0.17 & $\ldots$ & $\ldots$ & $\ldots$ & $\ldots$ \\
\hline & 1981 Jun 19 & 117 & $\mathrm{~B}$ & $\ldots$ & 0.20 & $\ldots$ & $\ldots$ & $\ldots$ & $\ldots$ \\
\hline & 1981 Aug 13 & 172 & $\mathrm{~B}$ & $\ldots$ & 0.30 & $\ldots$ & $\ldots$ & $\ldots$ & $\ldots$ \\
\hline & 1981 Nov 11 & 261 & $\mathrm{C}$ & $\ldots$ & 0.07 & $\ldots$ & $\ldots$ & $\ldots$ & $\ldots$ \\
\hline & 1982 Feb 27 & 369 & $\mathrm{~A}$ & $\ldots$ & 0.03 & $\ldots$ & $\ldots$ & $\ldots$ & $\ldots$ \\
\hline & 1982 Jun 25 & 489 & A & $\ldots$ & 0.07 & $\ldots$ & $\ldots$ & $\ldots$ & $\ldots$ \\
\hline & 1982 Oct 2 & 587 & $\mathrm{~A}$ & $\ldots$ & 0.07 & $\ldots$ & $\ldots$ & $\ldots$ & $\ldots$ \\
\hline & 1983 Feb 16 & 723 & $\mathrm{C}$ & $\ldots$ & 0.20 & $\ldots$ & $\ldots$ & $\ldots$ & $\ldots$ \\
\hline 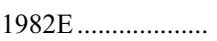 & 1985 Dec 29 & 1417 & $\mathrm{D}$ & 0.18 & 0.07 & $\ldots$ & $\ldots$ & $\ldots$ & $\ldots$ \\
\hline 1983G......................... & 1983 May 27 & 72 & $\mathrm{C}$ & $\ldots$ & 0.07 & $\ldots$ & $\ldots$ & $\ldots$ & $\ldots$ \\
\hline 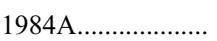 & 1984 Mar 5 & 430 & $\mathrm{BnC}$ & $\ldots$ & 0.10 & $\ldots$ & $\ldots$ & $\ldots$ & $\ldots$ \\
\hline \multirow{8}{*}{ 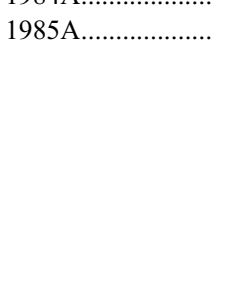 } & 1985 Feb 1 & 49 & A & $\ldots$ & 0.07 & $\ldots$ & $\ldots$ & $\ldots$ & $\ldots$ \\
\hline & $1985 \mathrm{Feb} 8$ & 56 & A & 0.07 & $\ldots$ & $\ldots$ & $\ldots$ & $\ldots$ & $\ldots$ \\
\hline & 1985 Feb 17 & 65 & A & 0.09 & 0.07 & $\ldots$ & $\ldots$ & $\ldots$ & $\ldots$ \\
\hline & 1985 Mar 2 & 81 & A & 0.07 & $\ldots$ & $\ldots$ & $\ldots$ & $\ldots$ & $\ldots$ \\
\hline & 1985 Apr 5 & 114 & $\mathrm{~A} / \mathrm{B}$ & 0.08 & $\ldots$ & $\ldots$ & $\ldots$ & $\ldots$ & $\ldots$ \\
\hline & 1985 Oct 28 & 320 & $\mathrm{C} / \mathrm{D}$ & 0.10 & $\ldots$ & $\ldots$ & $\ldots$ & $\ldots$ & $\ldots$ \\
\hline & 1986 Jun 15 & 550 & $\mathrm{~A} / \mathrm{B}$ & 0.24 & 0.14 & $\ldots$ & $\ldots$ & $\ldots$ & $\ldots$ \\
\hline & 1987 Oct 23 & 1045 & $\mathrm{~A} / \mathrm{B}$ & 0.26 & 0.09 & $\ldots$ & $\ldots$ & $\ldots$ & $\ldots$ \\
\hline \multirow[t]{5}{*}{ 1985B ............................ } & 1985 Feb 22 & 70 & A & 0.17 & 0.19 & $\ldots$ & $\ldots$ & $\ldots$ & $\ldots$ \\
\hline & 1985 Mar 18 & 94 & $\mathrm{~A} / \mathrm{B}$ & 0.40 & 0.06 & $\ldots$ & $\ldots$ & $\ldots$ & $\ldots$ \\
\hline & 1985 Sep 15 & 275 & $\mathrm{C}$ & 0.08 & 0.05 & $\ldots$ & $\ldots$ & $\ldots$ & $\ldots$ \\
\hline & 1986 Apr 30 & 502 & A & $\ldots$ & 0.16 & $\ldots$ & $\ldots$ & $\ldots$ & $\ldots$ \\
\hline & 1987 Sep 18 & 1008 & A & $\ldots$ & 0.07 & $\ldots$ & $\ldots$ & $\ldots$ & $\ldots$ \\
\hline \multirow[t]{7}{*}{ 1986A ........................ } & 1986 Feb 7 & 18 & $\mathrm{D}$ & $\ldots$ & 0.22 & $\ldots$ & $\ldots$ & $\ldots$ & $\ldots$ \\
\hline & 1986 Feb 25 & 36 & A & 0.36 & 0.12 & $\ldots$ & $\ldots$ & $\ldots$ & $\ldots$ \\
\hline & 1986 Mar 16 & 55 & A & $\ldots$ & 0.05 & $\ldots$ & $\ldots$ & $\ldots$ & $\ldots$ \\
\hline & 1986 Apr 3 & 73 & A & $\ldots$ & 0.09 & $\ldots$ & $\ldots$ & $\ldots$ & $\ldots$ \\
\hline & 1986 Jun 15 & 146 & $\mathrm{~A} / \mathrm{B}$ & 0.15 & 0.09 & $\ldots$ & $\ldots$ & $\ldots$ & $\ldots$ \\
\hline & 1986 Oct 16 & 269 & $\mathrm{~B} / \mathrm{C}$ & 0.25 & 0.08 & $\ldots$ & $\ldots$ & $\ldots$ & $\ldots$ \\
\hline & 1987 Apr 1 & 436 & $\mathrm{D}$ & $\ldots$ & 0.08 & $\ldots$ & 0.15 & $\ldots$ & $\ldots$ \\
\hline \multirow[t]{8}{*}{ 1986G........................ } & 1986 May 14 & 21 & A & $\ldots$ & 1.06 & $\ldots$ & $\ldots$ & $\ldots$ & $\ldots$ \\
\hline & 1986 May 21 & 28 & A & $\ldots$ & 0.70 & $\ldots$ & 3.21 & $\ldots$ & $\ldots$ \\
\hline & 1986 Jun 8 & 46 & $\mathrm{~A}$ & 7.48 & 1.02 & $\ldots$ & $\ldots$ & $\ldots$ & $\ldots$ \\
\hline & 1986 Jul 6 & 74 & $\mathrm{~A} / \mathrm{B}$ & $\ldots$ & 1.52 & $\cdots$ & 1.32 & $\cdots$ & $\cdots$ \\
\hline & 1986 Sep 20 & 150 & $\mathrm{C}$ & $\ldots$ & 2.49 & $\ldots$ & 1.27 & $\ldots$ & $\ldots$ \\
\hline & 1987 Jan 4 & 256 & $\mathrm{C}$ & $\ldots$ & 2.71 & $\ldots$ & 1.38 & $\ldots$ & $\ldots$ \\
\hline & 1987 Oct 23 & 548 & $\mathrm{~A} / \mathrm{B}$ & 5.06 & 1.31 & $\ldots$ & 4.47 & $\ldots$ & $\ldots$ \\
\hline & 1989 Apr 6 & 1079 & B & $\ldots$ & 4.08 & $\ldots$ & $\ldots$ & $\ldots$ & $\ldots$ \\
\hline \multirow[t]{6}{*}{$19860 \ldots \ldots \ldots \ldots \ldots \ldots$} & 1987 Feb 12 & 72 & $\mathrm{C} / \mathrm{D}$ & 0.80 & 0.07 & $\ldots$ & 0.18 & $\ldots$ & $\ldots$ \\
\hline & 1987 Apr 11 & 130 & $\mathrm{D}$ & $\ldots$ & 0.08 & $\ldots$ & 0.16 & $\ldots$ & $\ldots$ \\
\hline & 1987 May 24 & 173 & $\mathrm{D}$ & $\ldots$ & 0.08 & $\ldots$ & 0.15 & $\ldots$ & $\ldots$ \\
\hline & 1987 Aug 28 & 269 & A & $\ldots$ & 0.07 & $\ldots$ & $\ldots$ & $\ldots$ & $\ldots$ \\
\hline & 1988 Apr 3 & 488 & $\mathrm{C}$ & 0.49 & 0.06 & $\ldots$ & $\ldots$ & $\ldots$ & $\ldots$ \\
\hline & 1989 Jul 17 & 958 & $\mathrm{C}$ & $\ldots$ & 0.08 & $\ldots$ & $\ldots$ & $\ldots$ & $\ldots$ \\
\hline \multirow[t]{5}{*}{ 1987D ...................... } & 1987 May 15 & 46 & $\mathrm{D}$ & $\ldots$ & 0.20 & $\ldots$ & $\ldots$ & $\ldots$ & $\ldots$ \\
\hline & 1987 Jun 4 & 66 & $\mathrm{D}$ & $\ldots$ & 0.26 & $\ldots$ & $\ldots$ & $\ldots$ & $\ldots$ \\
\hline & 1987 Jun 21 & 83 & A & $\ldots$ & 0.06 & $\ldots$ & $\ldots$ & $\ldots$ & $\ldots$ \\
\hline & 1987 Sep 18 & 172 & A & $\ldots$ & 0.07 & $\ldots$ & $\ldots$ & $\ldots$ & $\ldots$ \\
\hline & 1988 May 29 & 426 & $\mathrm{C} / \mathrm{D}$ & $\ldots$ & 0.26 & $\ldots$ & $\ldots$ & $\ldots$ & $\ldots$ \\
\hline \multirow[t]{7}{*}{ 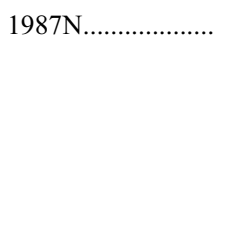 } & 1987 Dec 20 & 37 & $\mathrm{~B}$ & 0.13 & 0.10 & $\ldots$ & $\ldots$ & $\ldots$ & $\ldots$ \\
\hline & 1988 Jan 12 & 60 & $\mathrm{~B}$ & 0.10 & 0.08 & $\ldots$ & $\ldots$ & $\ldots$ & $\ldots$ \\
\hline & 1988 Feb 1 & 76 & $\mathrm{~B}$ & 0.11 & 0.09 & $\ldots$ & $\ldots$ & $\ldots$ & $\ldots$ \\
\hline & 1988 Mar 31 & 135 & $\mathrm{C}$ & 0.23 & 0.07 & $\ldots$ & $\ldots$ & $\ldots$ & $\ldots$ \\
\hline & 1988 Apr 11 & 146 & $\mathrm{C}$ & 0.22 & 0.40 & $\ldots$ & $\ldots$ & $\cdots$ & $\ldots$ \\
\hline & 1988 Aug 22 & 279 & $\mathrm{D}$ & $\ldots$ & 0.08 & $\ldots$ & $\ldots$ & $\cdots$ & $\cdots$ \\
\hline & 1989 Apr 24 & 524 & $\mathrm{~B}$ & $\ldots$ & 0.06 & $\ldots$ & $\ldots$ & $\ldots$ & $\ldots$ \\
\hline
\end{tabular}


TABLE 2 - Continued

\begin{tabular}{|c|c|c|c|c|c|c|c|c|c|}
\hline \multirow[b]{2}{*}{$\begin{array}{c}\mathrm{SN} \\
\text { NAME } \\
(1)\end{array}$} & \multirow[b]{2}{*}{$\begin{array}{c}\text { OBSERVATION } \\
\text { DATE } \\
(2)\end{array}$} & \multirow[b]{2}{*}{$\begin{array}{c}\mathrm{AGE}^{\mathrm{a}} \\
\text { (days) } \\
(3)\end{array}$} & \multirow[b]{2}{*}{$\begin{array}{l}\text { VLA } \\
\text { ConfIG. } \\
\quad(4)\end{array}$} & \multicolumn{6}{|c|}{ MAP rms $(1 \sigma)$} \\
\hline & & & & $\begin{array}{c}\sigma_{20} \\
(\mathrm{mJy}) \\
(5)\end{array}$ & $\begin{array}{c}\sigma_{6} \\
(\mathrm{mJy}) \\
(6)\end{array}$ & $\begin{array}{c}\sigma_{3.6} \\
(\mathrm{mJy}) \\
(7)\end{array}$ & $\begin{array}{c}\sigma_{2} \\
(\mathrm{mJy}) \\
(8)\end{array}$ & $\begin{array}{c}\sigma_{1.3} \\
(\mathrm{mJy}) \\
(9)\end{array}$ & $\begin{array}{c}\sigma_{0.7} \\
(\mathrm{mJy}) \\
(10)\end{array}$ \\
\hline \multirow[t]{9}{*}{ 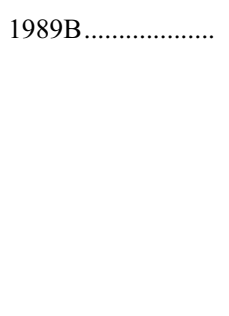 } & 1989 Feb $2^{\mathrm{b}}$ & 10 & $\mathrm{~A}$ & $\ldots$ & 0.08 & 0.06 & $\ldots$ & $\ldots$ & $\ldots$ \\
\hline & 1989 Feb 3 & 11 & $\mathrm{~A}$ & $\ldots$ & 0.03 & 0.03 & $\ldots$ & $\ldots$ & $\ldots$ \\
\hline & 1989 Mar 6 & 42 & $\mathrm{~A} / \mathrm{B}$ & $\ldots$ & 0.06 & $\ldots$ & $\ldots$ & $\ldots$ & $\ldots$ \\
\hline & 1989 Mar 27 & 63 & $\mathrm{~B}$ & $\ldots$ & 0.06 & $\ldots$ & $\ldots$ & $\ldots$ & $\ldots$ \\
\hline & 1989 Apr 6 & 73 & $\mathrm{~B}$ & $\ldots$ & 0.07 & $\ldots$ & $\ldots$ & $\ldots$ & $\ldots$ \\
\hline & 1989 May 15 & 112 & $\mathrm{~B} / \mathrm{C}$ & $\ldots$ & 0.06 & $\ldots$ & $\ldots$ & $\ldots$ & $\ldots$ \\
\hline & $1990 \mathrm{Jul} 22$ & 545 & B & $\ldots$ & $\ldots$ & 0.04 & $\ldots$ & $\ldots$ & $\ldots$ \\
\hline & 1993 Oct 25 & 1736 & $\mathrm{C} / \mathrm{D}$ & $\ldots$ & $\ldots$ & 0.04 & $\ldots$ & $\ldots$ & $\ldots$ \\
\hline & 2003 May 26 & 5236 & A & $\ldots$ & $\ldots$ & $\ldots$ & $\ldots$ & $\ldots$ & $\ldots$ \\
\hline \multirow[t]{6}{*}{ 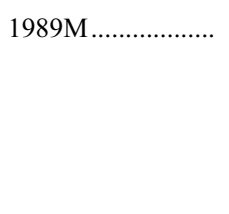 } & 1989 Jul 17 & 60 & $\mathrm{C}$ & $\ldots$ & 0.13 & $\ldots$ & $\ldots$ & $\ldots$ & $\ldots$ \\
\hline & 1989 Sep 4 & 109 & $\mathrm{C}$ & $\ldots$ & 0.10 & $\ldots$ & $\ldots$ & $\ldots$ & $\ldots$ \\
\hline & 1989 Oct 24 & 159 & $\mathrm{C} / \mathrm{D}$ & $\cdots$ & 0.07 & $\cdots$ & $\cdots$ & $\cdots$ & $\cdots$ \\
\hline & 1989 Dec 21 & 217 & $\mathrm{D}$ & $\cdots$ & 0.05 & $\cdots$ & $\cdots$ & $\cdots$ & $\cdots$ \\
\hline & 1990 Feb 13 & 271 & $\mathrm{D} / \mathrm{A}$ & $\ldots$ & 0.08 & $\ldots$ & $\ldots$ & $\ldots$ & $\ldots$ \\
\hline & 1990 May 29 & 376 & A & $\ldots$ & 0.15 & $\ldots$ & $\ldots$ & $\ldots$ & $\ldots$ \\
\hline \multirow[t]{2}{*}{ 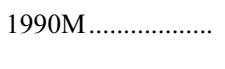 } & 1990 Jun 29 & 42 & $\mathrm{~A} / \mathrm{B}$ & $\ldots$ & $\ldots$ & 0.04 & $\ldots$ & $\ldots$ & $\ldots$ \\
\hline & 1990 Dec 14 & 210 & $\mathrm{C}$ & $\ldots$ & $\ldots$ & 0.04 & $\ldots$ & $\ldots$ & $\ldots$ \\
\hline \multirow[t]{3}{*}{$1991 \mathrm{~T}$} & 1991 May 8 & 24 & $\mathrm{D}$ & $\cdots$ & $\cdots$ & 0.05 & 0.06 & $\cdots$ & $\cdots$ \\
\hline & 1991 Jul 9 & 86 & $\mathrm{~A}$ & $\ldots$ & $\ldots$ & 0.06 & 0.20 & $\ldots$ & $\ldots$ \\
\hline & $1993 \mathrm{Feb} 2$ & 660 & $\mathrm{~A} / \mathrm{B}$ & 0.05 & $\ldots$ & 0.05 & $\ldots$ & $\ldots$ & $\ldots$ \\
\hline \multirow[t]{3}{*}{ 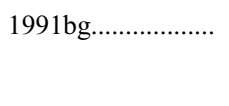 } & 1991 Dec 26 & 26 & $\mathrm{~B}$ & 0.26 & $\ldots$ & 0.16 & $\ldots$ & $\ldots$ & $\ldots$ \\
\hline & $1993 \mathrm{Feb} 2$ & 430 & $\mathrm{~A} / \mathrm{B}$ & $0.14^{\mathrm{c}}$ & $\ldots$ & 0.08 & $\ldots$ & $\ldots$ & $\ldots$ \\
\hline & 1993 Oct 25 & 695 & $\mathrm{C} / \mathrm{D}$ & $\ldots$ & $\ldots$ & $0.20^{\mathrm{b}}$ & $\ldots$ & $\ldots$ & $\ldots$ \\
\hline \multirow[t]{3}{*}{ 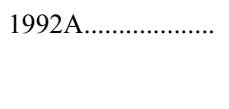 } & 1992 Jan 27 & 25 & $\mathrm{~B} / \mathrm{C}$ & $\ldots$ & 0.03 & $\ldots$ & $\ldots$ & $\ldots$ & $\ldots$ \\
\hline & 1992 Oct 9 & 281 & A & $\ldots$ & 0.15 & $\ldots$ & $\ldots$ & $\ldots$ & $\ldots$ \\
\hline & 1993 Feb 5 & 400 & $\mathrm{~A} / \mathrm{B}$ & $\ldots$ & 0.08 & $\ldots$ & $\ldots$ & $\ldots$ & $\ldots$ \\
\hline 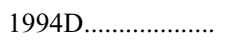 & 1994 May 4 & 57 & A & $\ldots$ & 0.06 & $\ldots$ & $\ldots$ & $\ldots$ & $\ldots$ \\
\hline 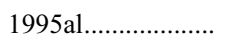 & 1995 Nov 8 & 13 & B & 0.08 & $\ldots$ & $\ldots$ & $\ldots$ & $\ldots$ & $\ldots$ \\
\hline 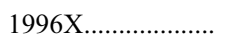 & 1996 May 29 & 55 & $\mathrm{DnC}$ & $\ldots$ & $\ldots$ & 0.09 & $\ldots$ & $\ldots$ & $\ldots$ \\
\hline \multirow[t]{4}{*}{ 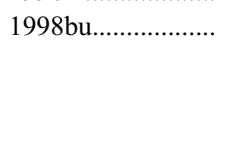 } & 1998 May 13 & 6 & A & $\ldots$ & $\ldots$ & 0.07 & $\ldots$ & $\ldots$ & $\ldots$ \\
\hline & 1998 May 31 & 24 & A & $\cdots$ & $\cdots$ & 0.04 & 0.18 & $\cdots$ & $\cdots$ \\
\hline & 1998 Jun 9 & 33 & AnB & $\ldots$ & $\ldots$ & 0.05 & 0.23 & $\ldots$ & $\ldots$ \\
\hline & 1999 Jan 7 & 245 & $\mathrm{C}$ & $\ldots$ & 0.07 & 0.06 & $\ldots$ & $\ldots$ & $\ldots$ \\
\hline \multirow[t]{2}{*}{ 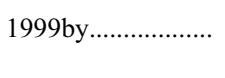 } & 1999 May 7 & 11 & $\mathrm{D}$ & $\cdots$ & $\ldots$ & 0.07 & $\cdots$ & $\cdots$ & $\cdots$ \\
\hline & 1999 May 24 & 28 & $\mathrm{D}$ & $\ldots$ & $\ldots$ & 0.08 & $\ldots$ & $\ldots$ & $\ldots$ \\
\hline \multirow{2}{*}{ 2002bo..................... } & 2002 May 21 & 73 & AnB & $\ldots$ & $\ldots$ & 0.06 & 0.28 & 0.36 & 0.62 \\
\hline & 2002 Jun 12 & 95 & B & 0.06 & 0.08 & 0.07 & $\ldots$ & $\ldots$ & $\ldots$ \\
\hline \multirow{2}{*}{$2002 \mathrm{cv} \ldots \ldots \ldots \ldots \ldots \ldots . . . . . .}$. & 2002 May 21 & 19 & $\mathrm{AnB}$ & $\ldots$ & $\ldots$ & 0.06 & 0.28 & 0.36 & 0.62 \\
\hline & 2002 Jun 12 & 41 & B & 0.06 & 0.08 & 0.07 & $\ldots$ & $\ldots$ & $\ldots$ \\
\hline 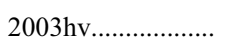 & 2003 Oct 21 & 57 & $\mathrm{~B}$ & $\ldots$ & $\ldots$ & 0.05 & $\ldots$ & $\ldots$ & $\ldots$ \\
\hline 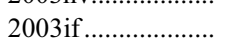 & 2003 Oct 21 & 64 & $\mathrm{~B}$ & $\cdots$ & $\cdots$ & 0.05 & $\cdots$ & $\cdots$ & $\cdots$ \\
\hline
\end{tabular}

a The explosion date is taken to be 18 days before the date of the optical maximum (Goldhaber et al. 2001).

${ }^{b}$ Observations were graciously contributed by R. Brown.

${ }^{\mathrm{c}}$ Severely confused by the southern radio lobe from the host galaxy, NGC 4374.

source of radio emission, consistent with the previous results from Weiler et al. $(1986,1989)$.

\section{RADIO LIGHT CURVE MODELING}

Following Weiler et al. (2002) and Sramek \& Weiler (2003), we adopt a parameterized model that, in view of the nondetection results, has been simplified to include only the intrinsic synchrotron emission from relativistic electrons created at the SN shock front, and possible free-free absorption by thermal electrons in a surrounding homogeneous CSM that has been ionized by the SN explosion itself. In this case, we can write

$$
S(\mathrm{mJy})=K_{1}\left(\frac{\nu}{5 \mathrm{GHz}}\right)^{\alpha}\left(\frac{t-t_{0}}{1 \text { day }}\right)^{\beta} e^{\tau_{\mathrm{CSM}, \text {,extemal }}}
$$

with

$$
\tau_{\mathrm{CSM}, \text { external }}=K_{2}\left(\frac{\nu}{5 \mathrm{GHz}}\right)^{-2.1}\left(\frac{t-t_{0}}{1 \text { day }}\right)^{\delta}
$$

where $K_{1}$ and $K_{2}$ correspond, formally but not necessarily physically, to the flux density $\left(K_{1}\right)$ and homogeneous $\left(K_{2}\right)$ absorption at $5 \mathrm{GHz}$ one day after the explosion date, $t_{0}$. The term $\tau_{\text {CSM.external }}$ is produced by an ionized medium that homogeneously covers the emitting source ("homogeneous external absorption"), and is near enough to the SN progenitor that it is altered by the rapidly expanding SN blast wave. The radial density $(\rho)$ distribution of this homogeneous external absorbing medium, if established by a constant mass-loss rate $(\dot{M})$, constant velocity $\left(w_{\text {wind }}\right)$, pre-SN stellar wind, is $r^{-2}$ [i.e., $\left.\rho \propto \dot{M} /\left(w_{\text {wind }} r^{2}\right)\right]$. The 


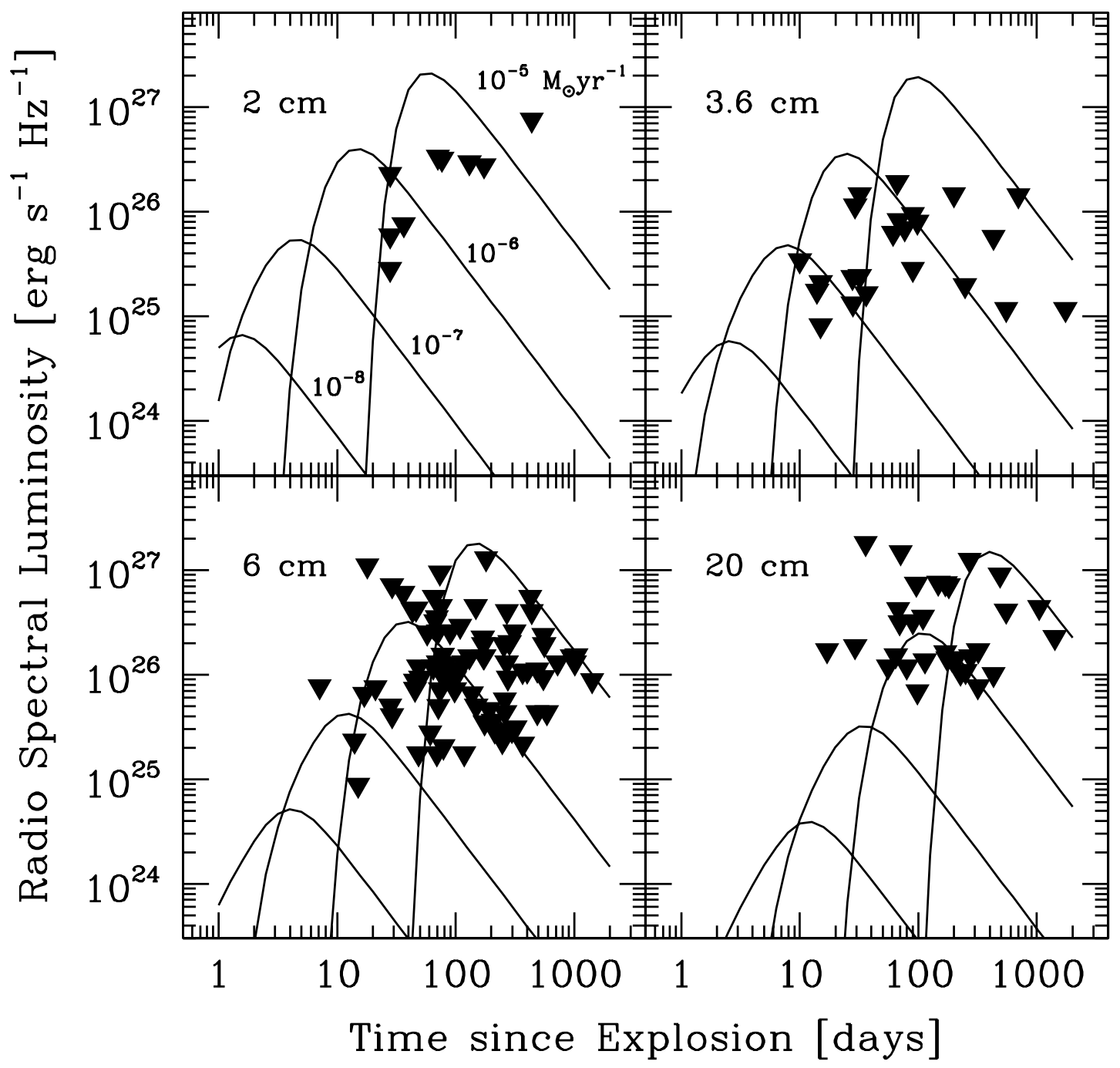

FIG. 1.- Upper limits $(2 \sigma)$ for all observed SNe Ia at 2, 3.6, 6, and $20 \mathrm{~cm}$ wavelength. Shown as examples are model radio light curves appropriate for SNe Ib/c (see text), assuming mass-loss rates associated with the progenitor systems of $10^{-8}, 10^{-7}, 10^{-6}$, and $10^{-5} M_{\odot} \mathrm{yr}^{-1}$ in a stellar wind with speed of $w_{\text {wind }}=10 \mathrm{~km} \mathrm{~s}{ }^{-1}$.

value of $\delta$ in equation (2) describes the actual radial density, if different from $r^{-2}$, for a constant shock velocity. The absorbing medium is assumed to be purely thermal singly ionized gas, which absorbs via free-free transitions with frequency dependence $\nu^{-2.1}$ in the radio.

Since the free-free optical depth outside the blast-wave-emitting region is proportional to the integral of the square of the CSM density over the radius, and in the simple model (Chevalier 1982a, 1982b) the CSM density decreases as $r^{-2}$, the external optical depth will be proportional to $r^{-3}$. With the blast wave radius increasing as a power of time, $r \propto t^{m}$ (with $m \leq 1$; i.e., $m=1$ for undecelerated blast wave expansion), it follows that the deceleration parameter, $m$, is

$$
m=-\delta / 3
$$

The success of the basic parameterization and modeling has been shown in the good correspondence between the model fits and the data for all RSN types, e.g., Type Ib SN 1983N (Sramek et al. 1984), Type Ic SN 1990B (Van Dyk et al. 1993a), Type II SN 1979C (Weiler et al. 1991, 1992b; Montes et al. 2000) and SN 1980K (Weiler et al. 1992a; Montes et al. 1998), and Type IIn SN 1988Z (Van Dyk et al. 1993b; Williams et al. 2002).
For the case of a steady pre-SN stellar wind, Weiler et al. $(1986,2001,2002)$ have shown that the mass-loss rate can be derived directly from the measured (free-free) optical depth as

$$
\begin{aligned}
& \frac{\dot{M} 1 M_{\odot} \mathrm{yr}^{-1}}{w_{\text {wind }} / 10 \mathrm{~km} \mathrm{~s}^{-1}}=3.0 \times 10^{-6}\left\langle\tau_{\text {eff }}^{0.5}\right\rangle m^{-1.5} \\
& \times\left(\frac{v_{i}}{10^{4} \mathrm{~km} \mathrm{~s}^{-1}}\right)^{1.5}\left(\frac{t_{i}}{45 \text { days }}\right)^{1.5}\left(\frac{t}{t_{i}}\right)^{1.5 m}\left(\frac{T}{10^{4} \mathrm{~K}}\right)^{0.68},
\end{aligned}
$$

where, since the appearance of optical lines for measuring SN ejecta velocities is often delayed a bit relative to the time of the explosion, they arbitrarily take $t_{i}=45$ days. Because observations have shown that generally $0.8 \leq m \leq 1.0$, and from equation (4) $\dot{M} \propto t_{i}^{1.5(1-m)}$, the dependence of the calculated mass-loss rate on the date $t_{i}$ of the initial ejecta velocity measurement is weak $\left(\dot{M} \propto t_{i}^{<0.3}\right)$, so that the best optical or VLBI velocity measurements available can be used without worrying about the deviation of the exact measurement epoch from the assumed 45 days after explosion. For convenience, and because many SN measurements indicate velocities of $\sim 10,000 \mathrm{~km} \mathrm{~s}^{-1}$, one usually assumes $v_{i}=v_{\text {blastwave }}=10,000 \mathrm{~km} \mathrm{~s}^{-1}, \mathrm{CSM}$ temperature $T=2 \times 10^{4} \mathrm{~K}$ 


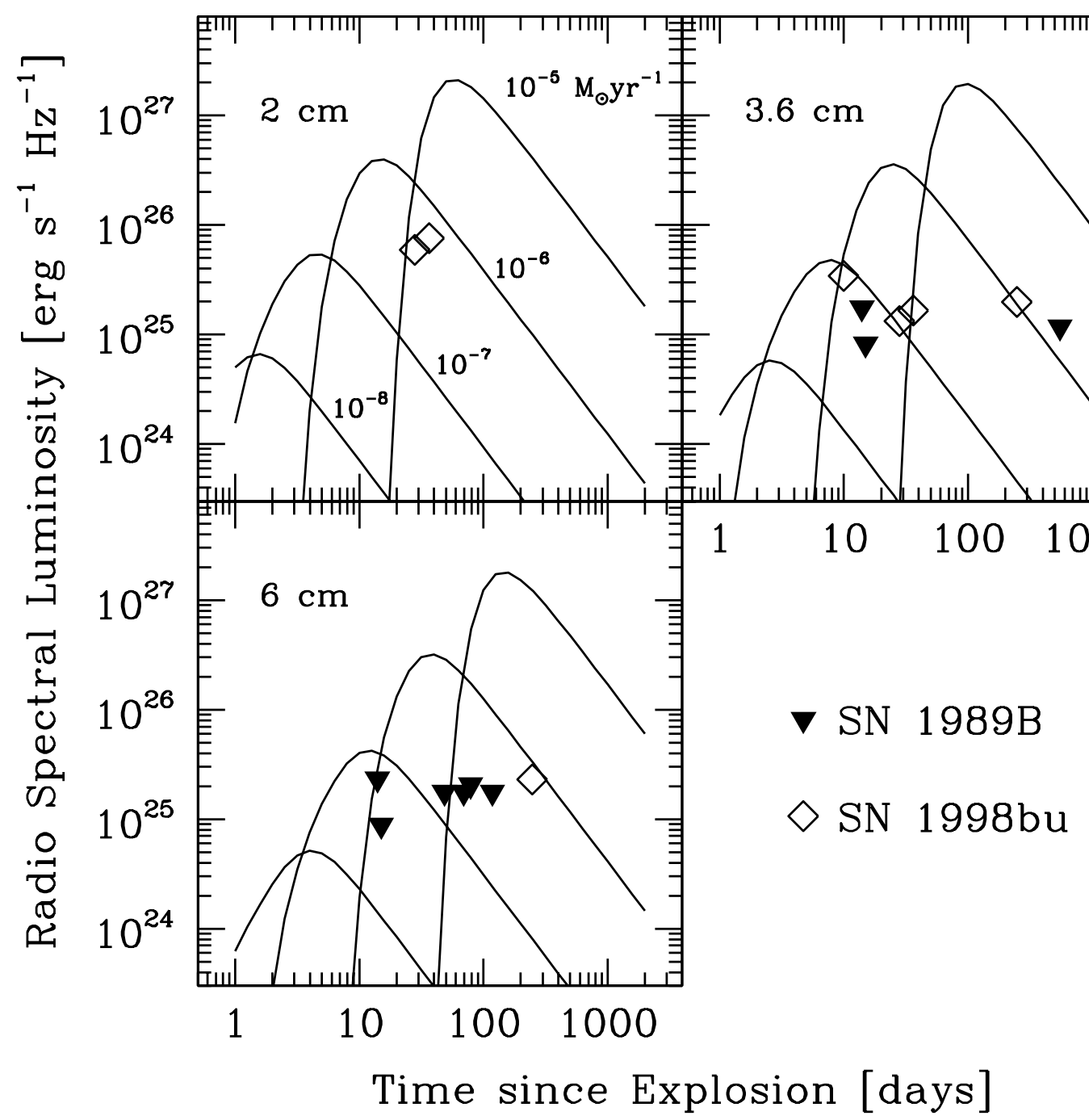

Fig. 2.- Upper limits ( $2 \sigma$ ) for SN 1989B at 2, 3.6, and $6 \mathrm{~cm}$ wavelength ( filled triangles) and SN 1998bu at 3.6 and $6 \mathrm{~cm}$ wavelength (open diamonds), compared with model radio light curves appropriate for $\mathrm{SNe} \mathrm{Ib/c} \mathrm{(see} \mathrm{text),} \mathrm{assuming} \mathrm{mass-loss} \mathrm{rates} \mathrm{associated} \mathrm{with} \mathrm{the} \mathrm{progenitor} \mathrm{systems} \mathrm{of} 10^{-8}, 10^{-7}, 10^{-6}$, and $10^{-5} M_{\odot} \mathrm{yr}^{-1}$ in a stellar wind with speed $w_{\text {wind }}=10 \mathrm{~km} \mathrm{~s}^{-1}$.

(appropriate for a red supergiant [RSG] wind), time $t=\left(t_{6} \mathrm{~cm}\right.$ peak $t_{0}$ ) days from best fits to the radio data for each RSN, and $m$ from equation (3).

The assumed pre-SN wind velocity, $w_{\text {wind }}=10 \mathrm{~km} \mathrm{~s}^{-1}$, appropriate for an RSG wind can also be generally applied to red giant companions in symbiotic systems and to recurrent novae (e.g., the CSM from the red giant wind in RS Oph is $\lesssim 20 \mathrm{~km} \mathrm{~s}^{-1}$; Hachisu \& Kato 2001). However, one should note that Shore et al. (1996) assume for RS Oph a red giant terminal velocity of 50$100 \mathrm{~km} \mathrm{~s}^{-1}$, and Solf et al. (1986) find bipolar winds in symbiotic systems with speeds in excess of $100 \mathrm{~km} \mathrm{~s}^{-1}$. For the case of possible dwarf and subdwarf winds, one would expect velocities of the order of their escape velocities, i.e., a few hundred $\mathrm{km} \mathrm{s}^{-1}$, but also much lower mass-loss rates. Therefore, in these cases, any mass transfer induced by stellar winds would have no effect on building up a dense CSM environment, and can safely be neglected in our discussion.

\section{APPLICATION TO TYPE Ia SUPERNOVAE}

Since the overall shape of the optical light curves of SNe Ia are rather similar to those of $\mathrm{SNe} \mathrm{Ib} / \mathrm{c}$ (although $\mathrm{SNe} \mathrm{Ib} / \mathrm{c}$ are generally $\sim 1-1.5$ mag fainter than SNe Ia; see, e.g., Leibundgut et al.
1991), suggesting comparable envelope masses and structures, we adopt for our analysis average parameters measured for $\mathrm{SNe} \mathrm{Ib/c}$ (see Weiler et al. 2002), namely $\alpha=-1.1, \beta=-1.5$, and $\delta=$ -2.6 . This is very similar to the approach taken by Boffi \& Branch (1995) who argued that, in particular for a symbiotic stellar system progenitor scenario, many of the parameters expected for $\mathrm{SNe}$ Ia (specifically, SN 1986G in their case) may be analogous to those for $\mathrm{SNe} \mathrm{Ib} / \mathrm{c}$.

As discussed earlier, the $\mathrm{SN}$ radio emission is a function of the CSM density, and hence is proportional to the ratio of the mass-loss rate to the wind velocity, $\dot{M} / w$. The theory developed by Chevalier (1982a, 1982b) provides a functional dependence between the intrinsic (i.e., before external free-free absorption by an ionized CSM) radio luminosity and the density of the CSM, of the form (Weiler et al. 1989, 2002)

$$
L_{\text {intrinsic }}(\nu) \propto(\dot{M} / w)^{(\gamma-7+12 m) / 4} m^{(5+\gamma) / 2} t^{-(\gamma+5-6 m) / 2} \nu^{-(\gamma-1) / 2},
$$

where

$$
\gamma=1-2 \alpha
$$


TABLE 3

Lowest Upper Limits to SN Ia Progenitor Mass-Loss Rates

\begin{tabular}{|c|c|c|c|c|c|}
\hline $\begin{array}{l}\text { SN } \\
\text { (1) }\end{array}$ & $\begin{array}{l}\text { Distance } \\
(\mathrm{Mpc}) \\
(2)\end{array}$ & $\begin{array}{c}\text { Epoch } \\
\text { (days) } \\
(3)\end{array}$ & $\begin{array}{l}\text { Wavelength } \\
\qquad(\mathrm{cm}) \\
(4)\end{array}$ & $\begin{array}{c}\text { Radio Luminosity }{ }^{\mathrm{a}} \\
\left(\mathrm{ergs}^{-1} \mathrm{~Hz}^{-1}\right) \\
\text { (5) }\end{array}$ & $\begin{array}{c}\dot{M}^{\mathrm{b}} \\
\left(M_{\odot} \mathrm{yr}^{-1}\right) \\
(6)\end{array}$ \\
\hline $1980 \mathrm{~N}$ & 23.3 & 71 & 6 & $2.5 \times 10^{26}$ & $1.1 \times 10^{-6}$ \\
\hline 1981B …................. & 16.6 & 17 & 6 & $6.5 \times 10^{25}$ & $1.3 \times 10^{-7}$ \\
\hline 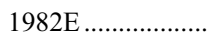 & 23.1 & 1416 & 20 & $2.3 \times 10^{26}$ & $7.3 \times 10^{-6}$ \\
\hline 1983G..................... & 17.8 & 71 & 6 & $5.0 \times 10^{25}$ & $4.1 \times 10^{-7}$ \\
\hline 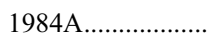 & 17.4 & 74 & 6 & $7.1 \times 10^{25}$ & $5.3 \times 10^{-7}$ \\
\hline 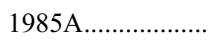 & 26.8 & 55 & 20 & $1.2 \times 10^{26}$ & $2.5 \times 10^{-7}$ \\
\hline 1985B ..................... & 28.0 & 69 & 20 & $3.1 \times 10^{26}$ & $6.1 \times 10^{-7}$ \\
\hline 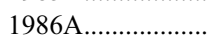 & 46.1 & 57 & 6 & $2.6 \times 10^{26}$ & $9.2 \times 10^{-7}$ \\
\hline 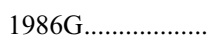 & 5.5 & 28 & 6 & $5.0 \times 10^{25}$ & $1.7 \times 10^{-7}$ \\
\hline 19860........................ & 28 & 71 & 6 & $1.3 \times 10^{26}$ & $7.4 \times 10^{-7}$ \\
\hline 1987D ......................... & 30 & 83 & 6 & $1.3 \times 10^{26}$ & $8.4 \times 10^{-7}$ \\
\hline 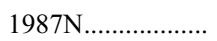 & 37.0 & 67 & 20 & $4.2 \times 10^{26}$ & $7.4 \times 10^{-7}$ \\
\hline 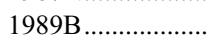 & 11.1 & 15 & 3.6 & $8.1 \times 10^{24}$ & $3.3 \times 10^{-8}$ \\
\hline 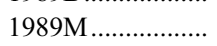 & 17.4 & 50 & 6 & $9.2 \times 10^{25}$ & $4.4 \times 10^{-7}$ \\
\hline 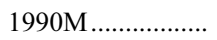 & 39.4 & 32 & 3.6 & $1.5 \times 10^{26}$ & $5.4 \times 10^{-7}$ \\
\hline 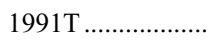 & 14.1 & 28 & 3.6 & $2.3 \times 10^{25}$ & $1.5 \times 10^{-7}$ \\
\hline 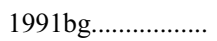 & 17.4 & 29 & 3.6 & $1.1 \times 10^{26}$ & $2.0 \times 10^{-7}$ \\
\hline 1992A ………......... & 24.0 & 29 & 6 & $4.1 \times 10^{25}$ & $1.6 \times 10^{-7}$ \\
\hline 1994D ………… & 14 & 61 & 6 & $2.8 \times 10^{25}$ & $2.5 \times 10^{-7}$ \\
\hline 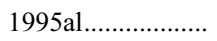 & 30 & 17 & 20 & $1.7 \times 10^{26}$ & $1.2 \times 10^{-7}$ \\
\hline 1996X & 30 & 66 & 3.6 & $1.9 \times 10^{26}$ & $1.2 \times 10^{-6}$ \\
\hline 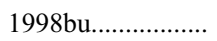 & 11.8 & 28 & 3.6 & $1.3 \times 10^{25}$ & $1.1 \times 10^{-7}$ \\
\hline 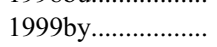 & 11.3 & 15 & 3.6 & $2.1 \times 10^{25}$ & $8.0 \times 10^{-8}$ \\
\hline 2002bo............................ & 22 & 95 & 20 & $6.8 \times 10^{25}$ & $3.0 \times 10^{-7}$ \\
\hline 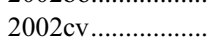 & 22 & 41 & 20 & $6.8 \times 10^{25}$ & $3.0 \times 10^{-7}$ \\
\hline 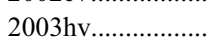 & 23 & 61 & 3.6 & $6.2 \times 10^{25}$ & $5.8 \times 10^{-7}$ \\
\hline 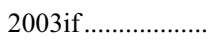 & 26.4 & 68 & 3.6 & $8.1 \times 10^{25}$ & $7.6 \times 10^{-7}$ \\
\hline
\end{tabular}

${ }^{a}$ The spectral luminosity upper limit $(2 \sigma)$, as estimated at the wavelength given in col. (4), which, when combined with the age of the SN at the time of observation, yielded the lowest mass-loss rate limit.

b The upper limit $(2 \sigma)$ to the mass-loss rate, $\dot{M}$, is calculated from the spectral luminosity lowest upper limit given in col. (5), as measured at the wavelength given in col. (4) at an epoch after explosion given in col. (3). The mass-loss limits are calculated with the assumption that the SN Ia progenitor systems can be modeled by the known properties of $\mathrm{SN} \mathrm{Ib} / \mathrm{c}$ progenitor systems, and that the pre-SN wind velocity establishing the CSM is $w_{\text {wind }}=10 \mathrm{~km} \mathrm{~s}^{-1}$.

For the assumed $\alpha=-1.1(\gamma=3.2), \delta=-2.6(m=-\delta / 3=$ $0.87)$, and at $\nu=5 \mathrm{GHz}$, this becomes

$$
L_{\text {intrinsic }}(\nu) \propto(\dot{M} / w)^{1.65} t^{-1.5} \nu^{-1.1}
$$

Multiplying by the attenuation produced by external (freefree) absorption, it becomes analogous to equation (1), but when expressed in absolute units is

$$
\begin{aligned}
\frac{L}{10^{26} \mathrm{erg} \mathrm{s}^{-1} \mathrm{~Hz}^{-1}}= & \Lambda\left(\frac{\dot{M} 1 M_{\odot} \mathrm{yr}^{-1}}{w_{\text {wind }} / 10 \mathrm{~km} \mathrm{~s}^{-1}}\right)^{1.65} \\
& \times\left(\frac{\nu}{5 \mathrm{GHz}}\right)^{-1.1}\left(\frac{t-t_{0}}{1 \text { day }}\right)^{-1.5} e^{-\tau_{\mathrm{CSM}_{\text {external }}}}
\end{aligned}
$$

The parameter $\Lambda$ is a proportionality constant which is not provided by theory and must be calibrated empirically from radio observations of $\mathrm{SNe} \mathrm{Ib} / \mathrm{c}$. Since we are dealing with sources that are intrinsically faint, we calibrate $\Lambda$ using the best measured faint SN Ib/c, namely, SN 1983N (Sramek et al. 1984; Weiler et al. 1989, 2002), obtaining

$$
\Lambda=1285 \pm 245
$$

The quoted error corresponds to the combination in quadrature of the uncertainty of the light curve fit $(\sim 14 \%)$ as given by Weiler et al. (1986) and an estimated uncertainty in the distance to M83 of $\sim 7 \%$ (Thim et al. 2003). Also, one should be aware that appreciably different values of $\Lambda$ would be obtained from using different $\mathrm{SNe} \mathrm{Ib} / \mathrm{c}$ listed in Table 3 of Weiler et al. (2002) for its estimation, with the bright $\mathrm{SNe}$ providing systematically lower values than the fainter ones, with a range of a factor of 10 between the extremes. However, because of the functional dependence of $L$ on $\dot{M} / w_{\text {wind }}$, such a spread would result in an overall uncertainty in $\dot{M}$ of at most a factor of 2 .

For each $\mathrm{SN}$, the upper limits to the mass-loss rates were estimated by direct comparisons of the radio luminosity upper limits with a set of theoretical light curves calculated for all relevant epochs, and for values of the parameter $\dot{M} / w_{\text {wind }}$ between $10^{-9}$ and $10^{-6} M_{\odot} \mathrm{yr}^{-1} \mathrm{~km}^{-1} \mathrm{~s}$ at logarithmic steps of 0.05 . In the case of SNe with observations at different frequencies, the overall upper limit to $\dot{M} / w_{\text {wind }}$ was taken as the minimum value among those determined for each frequency. Finally, the massloss rates were calculated assuming the wind velocity to be $w=$ $10 \mathrm{~km} \mathrm{~s}^{-1}$, as appropriate for winds from red giants and RSGs, as well as from binary systems with total mass of a few solar masses and separations of a few AU.

Table 3 lists upper limits to the mass-loss rates from the SN Ia progenitor systems. Column (1) lists the SN name, and column (2) its distance taken from direct Cepheid determinations, whenever 
possible, or from the Revised Shapley-Ames Catalog of Bright Galaxies (Sandage \& Tammann 1987), rescaled to a value of the Hubble constant $H_{0}=63 \mathrm{~km} \mathrm{~s}^{-1} \mathrm{Mpc}^{-1}$ (Panagia 2003). Columns (3)-(5) list the epoch, the wavelength, and the $2 \sigma$ limit to the spectral luminosity that yielded the lowest mass-loss rate limit, and column (6) lists the $2 \sigma$ limit to the mass-loss rate, $\dot{M}$.

\section{DISCUSSION}

Examination of Table 3 shows that our upper limits are generally consistent with pre-SN mass-loss rates lower than $10^{-6} M_{\odot} \mathrm{yr}^{-1}$ associated with the progenitor system, with almost half (actually 12 out of 27) of the $2 \sigma$ limits falling below $\sim 4 \times 10^{-7} M_{\odot} \mathrm{yr}^{-1}$. In particular, the most stringent upper limits are provided by the observations of SNe 1989B and 1998bu, as shown in Figure 2. Not surprisingly, it also appears that most of the lowest upper limits are found for relatively nearby SNe Ia observed at early epochs, e.g., $\sim 10-50$ days after explosion or about -10 to +30 days relative to the optical maximum, and at frequencies $5-8.3 \mathrm{GHz}(\lambda=$ $3.6-6 \mathrm{~cm})$. This is because the VLA sensitivity is highest in the 5 and $8.3 \mathrm{GHz}$ bands, and the expected radio light curves at these frequencies peak around epochs of 10 days for mass-loss rates of $\sim 10^{-7} M_{\odot} \mathrm{yr}^{-1}$. On this basis, we argue that future searches should focus their efforts in the 5 and $8.3 \mathrm{GHz}$ bands, making prompt observations of SNe Ia soon after they are discovered, and repeating the observations about every 10 days until one or two months past the optical maximum. Additional observations at later times and at longer wavelengths would also be useful to check the possibility that a nondetection may be due to strong (free-free) absorption (i.e., indicating a high mass-loss rate) rather than merely to intrinsically faint emission.

We can derive a more stringent limit if we assume that all $\mathrm{SNe}$ Ia have the same type of progenitors and that they reach the explosion along identical evolutionary paths. In this case, we can combine the upper limits derived for individual objects, because they could be considered as the "errors" in a series of independent measurements, i.e., $\sigma_{\text {combined }}^{2}=1 / \Sigma_{i}\left(1 / \sigma_{i}^{2}\right)$. In this way, we obtain a combined $2 \sigma$ upper limit of $\lesssim 2.6 \times 10^{-8} M_{\odot} \mathrm{yr}^{-1}$.

Such low mass-loss rates as we have estimated generally rule out any red giant or red supergiant SN progenitors (consistent with current theories), and also exclude any SN Ia progenitor models that invoke a stellar wind from a (massive) companion to provide the required accretion rate onto a WD. Nomoto et al. (1984) calculated that only accretion rates appreciably greater than $\sim 4 \times 10^{-8} M_{\odot} \mathrm{yr}^{-1}$ can lead to a mass increase of the accreting WD resulting in a SN Ia explosion. More recently, Prialnik \& Kovetz (1995) estimated that accretion rates $\gtrsim 10^{-7} M_{\odot} \mathrm{yr}^{-1}$ are needed for a WD to increase its mass so as to exceed the Chandrasekhar mass.

Since accretion from a companion star's wind is a rather inefficient process, with $\sim 10 \%$ of the wind material being accreted onto a WD (Yungelson et al. 1995), only stellar companions with mass-loss rates $\gtrsim 4-10 \times 10^{-7} M_{\odot} \mathrm{yr}^{-1}$ would be able to satisfy the requirement. These $\dot{M}$ values are much greater $(\sim 10-$ 25 times) than the best individual, and particularly the aggregate, upper limits derived from our SN Ia radio observations. Thus, we support the conclusion reached by Boffi \& Branch (1995) and Eck et al. (1995) that symbiotic systems are unlikely to be SN progenitors.

An alternative to wind accretion onto the WD is Roche lobe overflow from a giant, subgiant, or main-sequence companion (see Branch et al. 1995; Livio 2001), so that the process is more gradual and efficient. In this case, our best combined upper limit, i.e., $2.6 \times 10^{-8} M_{\odot} \mathrm{yr}^{-1}$, implies that such a mass transfer must have an efficiency of $\gtrsim 60 \%-80 \%$ (depending on the adopted limiting accretion rate for WDs to become SNe Ia) to avoid leaving a residual CSM from which synchrotron emission would be detectable at current radio limits.

Double-degenerate models for SN Ia events in which the explosion is triggered by the merger of two WDs in a binary system are, of course, still consistent with our observations.

One should keep in mind that our mass-loss rate estimates are dependent on the assumptions that SN Ia radio light curves will be very similar to those of SNe Ib/c, and that $w_{\text {wind }}=10 \mathrm{~km} \mathrm{~s}^{-1}$, $v_{i}=10^{4} \mathrm{~km} \mathrm{~s}^{-1}$, and $T=2 \times 10^{4} \mathrm{~K}$, with dependences shown in equations (4) and (8). Even if higher values for any of these quantities are more appropriate or realistic, and will therefore yield higher mass-loss limits and correspondingly less stringent limits on the properties of the progenitor systems, we can definitely rule out the symbiotic system scenario based on our complete data set.

\section{CONCLUSIONS}

No radio emission has been found from $27 \mathrm{SNe}$ Ia observed over two decades with the VLA. It is clear that the CSM environment is far more tenuous than that of SNe II, or even $\mathrm{SNe} \mathrm{Ib} / \mathrm{c}$. Using model predictions of radio emission from $\mathrm{SNe}$ and assuming that the radio properties of SNe Ia would be relatively similar to those of $\mathrm{SNe} \mathrm{Ib} / \mathrm{c}$ (at least for some progenitor system models), but with a wind-established CSM from a less massive pre-SN system, we can place constraints on the mass-loss rate from such systems. If $\mathrm{SNe}$ Ia originate from mass accretion from a massive companion's wind onto a WD, we can establish a stringent limit of $\dot{M} \lesssim 3 \times 10^{-8} M_{\odot} \mathrm{yr}^{-1}$ in the best cases. This severely limits the possibility that the progenitors are symbiotic systems, where the companion is a red giant or supergiant star. However, highefficiency mass transfer through Roche lobe overflow from a lower mass companion or the double degenerate scenario, involving the merger of two WDs or a WD and a neutron star, cannot be ruled out.

We note that even in the most unusual case so far, of the recent SN Ia 2002ic, which exhibited optical evidence for a substantial CSM (Hamuy et al. 2003), it was concluded that the doubledegenerate scenario was the most consistent model for the progenitor of that event (Hamuy et al. 2003; Livio \& Riess 2003). Attempts to detect radio emission from SN 2002ic with the VLA were unsuccessful, likely due to the SN's large distance (Stockdale et al. 2003; Berger et al. 2003). Another recent example appears to be SN 2005gj (Prieto et al. 2005), which also has not been detected using the VLA (Soderberg \& Frail 2005). We further note, of course, that such events are likely intrinsically quite rare in general.

Clearly, additional observations of new SNe Ia should be made with the VLA as early and as deeply after discovery as possible, to help further constrain the nature of SN Ia progenitors. We continue to attempt observations such as these. However, with the current VLA, based on the results presented in this paper, realistically only the next Local Group SN Ia, or possibly a SN in a nearby galaxy group, will provide greatly improved upper limits. The plans for the Expanded VLA, ${ }^{9}$ and the far greater sensitivity that the upgrade will produce, will likely soon afford us with an unprecedented opportunity to detect radio emission from $\mathrm{SNe}$ Ia, and help define their progenitor systems. Considering the cosmological implications, such future observations are essential.

\footnotetext{
${ }^{9}$ See http://www.nrao.edu/evla/.
} 
K. W. W. thanks the Office of Naval Research for the 6.1 funding supporting this research. C. J. S. is a Cottrell Scholar of Research Corporation and work on this project has been sup- ported by the NASA Wisconsin Space Grant Consortium. Additional information and data on RSNe can be found at http://rsdwww.nrl.navy.mil/7210/7213/index.htm and linked pages.
Berger, E., Soderberg, A. M., \& Frail, D. A. IAU Circ., 8157, 2

Boffi, F. R., \& Branch, D. 1995, PASP, 107, 347

Branch, D., Doggett, J. B., Nomoto, K., \& Thielemann, F.-K. 1985, ApJ, 294, 619

Branch, D., Livio, M., Yungelson, L. R., Boffi, F. R., \& Baron, E. 1995, PASP, 107,1019

Chevalier, R. A. 1982a, ApJ, 259, 302 1982b, ApJ, 259, L85

Cowan, J. J., \& Branch, D. 1982, ApJ, 258, 31 1985, ApJ, 293, 400

Di Paola, A., et al. 2002, A\&A, 393, L21

Eck, C. R., Cowan, J. J., Roberts, D. A., Boffi, F. R., \& Branch, D. 1995, ApJ, 451, L53

Filippenko, A. V., et al. 1992a, ApJ, 384, L15 1992b, AJ, 104, 1543

Goldhaber, G., et al. 2001, ApJ, 558, 359

Hachisu, I., \& Kato, M. 2001, ApJ, 558, 323

Hamuy, M., et al. 2003, Nature, 424, 651

Iben, I., \& Tutukov, A. V. 1984, ApJS, 54, 335

Kirshner, R. P., et al. 1993, ApJ, 415, 589

Leibundgut, B., Tammann, G. A., Cadonau, R., \& Cerrito, D. 1991, A\&AS, 89, 537

Leibundgut, B., et al. 1993, AJ, 105, 301

Livio, M. 2001, in Supernovae and Gamma-Ray Bursts, ed. M. Livio, N. Panagia, \& K. Sahu (Cambridge: Cambridge Univ. Press), 334

Livio, M., \& Riess, A. G. 2003, ApJ, 594, L93

Montes, M. J., Van Dyk, S. D., Weiler, K. W., Sramek, R. A., \& Panagia, N. 1998, ApJ, 506, 874

Montes, M. J., Weiler, K. W., Van Dyk, S. D., Sramek, R. A., Panagia, N., \& Park, R. 2000, ApJ, 532, 1124

Nomoto, K., Thielemann, F., \& Yokoi, K. 1984, ApJ, 286, 644

Panagia, N. 2005, in IAU Colloq. 192, Cosmic Explosions, ed. J. M. Marcaide \& K. W. Weiler (Berlin: Springer), 585

Perlmutter, S., \& Schmidt, B. P. 2003, in Supernovae and Gamma-Ray Bursters, ed. K. W. Weiler (Berlin: Springer), 195

Perlmutter, S., et al. 1999, ApJ, 517, 565

Phillips, M. M., et al. 1987, PASP, 99, 592

Prialnik, D., \& Kovetz, A. 1995, ApJ, 445, 789

\section{REFERENCES}

Prieto, J., et al. 2005, IAU Circ., 8633, 1

Richmond, M. W., et al. 1995, AJ, 109, 2121

Riess, A. G., et al. 1998, AJ, 116, 1009

Ritter, H., \& Burkert, A. 1986, A\&A, 158, 161

Sandage, A., \& Tammann, G. A. 1987, Revised Shapley-Ames Catalog of Bright Galaxies (2nd ed.; Washington: Carnegie Inst.)

Schmidt, B. P., Kirshner, R. P., Leibundgut, B., Wells, L. A., Porter, A. C., Ruiz-Lapuente, P., Challis, P., \& Filippenko, A. V. 1994, ApJ, 434, L19

Shore, S. N., Kenyon, S. J., Starrfield, S., \& Sonneborn, G. 1996, ApJ, 456, 717

Soderberg, A. M., \& Frail, D. A. 2005, ATel, 663, 1

Solf, J., Böhm, K.-H., \& Raga, A. C. 1986, ApJ, 305, 795

Sramek, R. A., Panagia, N., \& Weiler, K. W. 1984, ApJ, 285, L59

Sramek, R. A., \& Weiler, K. W. 2003, in Supernovae and Gamma-Ray Bursters, ed. K. W. Weiler (Berlin: Springer), 145

Stockdale, C. J., et al. 2003, IAU Circ., 8157, 3

Szabo, Gy. M., et al. 2003, A\&A, 408, 915

Thim, F., Tammann, G. A., Saha, A., \& Labhardt, L. 2003, ApJ, 590, 256

Van Dyk, S., Sramek, R. A., Weiler, K., \& Panagia, N. 1993a, ApJ, 419, L69 1993b, ApJ, 409, 162

Webbink, R. F. 1984, ApJ, 277, 355

Weiler, K. W., Panagia, N., \& Montes, M. J. 2001, ApJ, 562, 670

Weiler, K. W., Panagia, N., Montes, M. J., \& Sramek, R. A. 2002, ARA\&A, 40, 387

Weiler, K. W., Panagia, N., \& Sramek, R. A. 1990, ApJ, 364, 611

Weiler, K. W., Panagia, N., Sramek, R. A., van der Hulst, J. M., Roberts, M. S., \& Nguyen, L. 1989, ApJ, 336, 421

Weiler, K. W., \& Sramek, R. A. 1988, ARA\&A, 26, 295

Weiler, K. W., Sramek, R. A., Panagia, N., van der Hulst, J. M., \& Salvati, M. 1986, ApJ, 301, 790

Weiler, K. W., Van Dyk, S. D., Discenna, J. L., Panagia, N., \& Sramek, R. A 1991, ApJ, 380, 161

Weiler, K. W., Van Dyk, S. D., Panagia, N., \& Sramek, R. A. 1992a, ApJ, 398, 248

Weiler, K. W., Van Dyk, S. D., Pringle, J., \& Panagia, N. 1992b, ApJ, 399, 672 Williams, C. L., Panagia, N., Van Dyk, S. D., Lacey, C. K., Weiler, K. W., \& Sramek, R. A. 2002, ApJ, 581, 396

Yungelson, L., Livio, M., Tututkov, A., \& Kenyon, S. J. 1995, ApJ, 447, 656 